\title{
Glucosylsphingosine Accumulation in Mice and Patients with Type 2 Gaucher Disease Begins Early in Gestation
}

\author{
EDUARD ORVISKY, ELLEN SIDRANSKY, CINDY E. MCKINNEY, MARY E. LAMARCA, \\ ROXANA SAMIMI, DONNA KRASNEWICH, BRIAN M. MARTIN, AND EDWARD I. GINNS \\ Clinical Neuroscience Branch, National Institute of Mental Health, National Institutes of Health, \\ Bethesda, Maryland 20892, U.S.A.
}

\begin{abstract}
Gaucher disease, the most common of the sphingolipidoses, results from the inherited deficiency of the enzyme glucocerebrosidase (EC 3.2.1.45). Although type 2 (acute neuronopathic) Gaucher disease is associated with rapidly progressive and fatal neurologic deterioration, the pathophysiologic mechanisms leading to the neurologic symptoms and early demise remain uncharacterized. While the pathology encountered in Gaucher disease has been attributed to glucocerebroside storage, glucosylsphingosine (Glc-sph), a cytotoxic compound, also accumulates in the tissues. Elevations of brain Glc-sph have been reported in patients with types 2 and 3 Gaucher disease. In this study, Glc-sph levels were measured using HPLC in tissues from mice with type 2 Gaucher disease created with a null glucocerebrosidase allele. Compared with unaffected littermates, homozygous mice with type 2 Gaucher disease had approximately a 100-fold elevation of Glc-sph in brain, as well as elevated levels in other tissues.
\end{abstract}

\section{ABSTRACT}

This accumulation was detected in utero by E 13 and increased progressively throughout gestation. Similarly, elevated Glc-sph levels were seen in human fetuses with type 2 Gaucher disease, indicating that therapy initiated after birth may be too late to prevent the sequaelae of progressive neurologic damage that begins early in gestation. These findings suggest that the accumulation of Glc-sph may be responsible for the rapid demise of mice with type 2 Gaucher disease and the devastating clinical course seen in patients with type 2 Gaucher disease. (Pediatr Res 48: 233-237, 2000)

Abbreviations
NBD-F, 4-fluoro-7-nitrobenzofurazan
Glc-sph, glucosylsphingosine
Gal-sph, galactosylsphingosine
NeoR, neomycin resistance gene

Gaucher disease (MIM \#230800), the inherited deficiency of the lysosomal enzyme glucocerebrosidase (EC 3.2.1.45), manifests with diverse clinical symptoms, including progressive neurologic deterioration. Gaucher disease was first described in 1882 by Gaucher (1). It has long been accepted that the accumulation of glucocerebroside in reticuloendothelial cells underlies the pathogenesis of Gaucher disease, transforming macrophages throughout the reticuloendothelial system into characteristic Gaucher cells, the hallmark pathologic finding of the disease $(2,3)$. However, the pathophysiologic mechanism leading to the neurologic symptoms seen in this disorder remains unknown (4-6). Glucocerebroside, which is widely distributed in cellular membranes, is released during the turnover of senescent white and red blood cell membranes, and is a metabolic intermediate in both the synthesis and degradation

Received December 7, 1999; accepted March 29, 2000.

Correspondence: Ellen Sidransky, M.D., Clinical Neuroscience Branch, National Institute of Mental Health, Building 49, Room B1EE16, 49 Convent Drive, MSC4405, Bethesda, MD 20892-4405, U.S.A.

Edward Orvisky and Ellen Sidransky contibuted equally to this work.

Supported by Fogarty International during fellowship (E.O.). of complex glycosphingolipids, including gangliosides and globoside. Glucocerebroside is predominantly degraded within lysosomes by glucocerebrosidase to glucose and ceramide, the latter being further degraded by ceramidase to sphingosine and fatty acids $(7,8)$. The accumulation of glucocerebroside in the liver, spleen, and bone marrow contributes to the massive organ enlargement and decreased red blood cells and platelets, as well as the skeletal deterioration, seen in patients with Gaucher disease.

Type 2, or acute neuronopathic Gaucher disease (MIM \#230900), is the most rare and severe form of Gaucher disease, usually resulting in death in infancy $(9,10)$. The devastating neurologic deterioration seen in patients with type 2 Gaucher disease has been attributed to the accumulation of glucocerebroside in the brain. However, many aspects of the pathology encountered in Gaucher disease are not well explained solely by the storage of glucocerebroside. For example, the amount of glucocerebroside in liver samples from patients with Gaucher disease is not sufficient to account for the degree of hepatomegaly on the basis of mass effect. Likewise, the relatively limited CNS pathology and storage found in patients and mice 
with type 2 Gaucher disease as compared to the rapidly progressive neurologic deterioration observed (11). Neither the amount of stored lipid nor the level of the residual glucocerebrosidase activity correlate well with patient phenotypes (12). Even the relationship between genotype and phenotype in patients with Gaucher disease is complex, and it is often difficult to correlate specific symptoms with a unique genotype $(13,14)$.

The enzyme glucocerebrosidase also degrades another substrate, Glc-sph, to glucose and sphingosine. Patients with both type 2 and 3 Gaucher disease accumulate Glc-sph in tissues, including the cerebral and cerebellar cortex (15). Nilsson and Svennerholm (15) suggested that this toxic substance, also known as glucosylpsychosine, could be responsible for the neuropathology seen in Gaucher disease. Moreover, it has been suggested that Glc-sph can disrupt normal cell activity by interfering with signal transduction and cellular differentiation (16), and may be involved in programmed cell death (17).

A murine model of type 2 Gaucher disease with a null allele was created by targeted homologous recombination to enable a better understanding of the pathophysiology of this disorder (18). For this model, portions of exons 9 and 10 of the murine glucocerebrosidase gene were removed and replaced with a selectable neomycin marker. Homozygous mice with type 2 Gaucher disease accumulate glucocerebroside in tissues, particularly within lysosomes of macrophages, as demonstrated by both thin layer chromatography and electron microscopy (19, 20). A pathologic examination of the nervous system, performed in an attempt to explain the rapid postnatal death of the mice and patients severely affected with type 2 Gaucher disease, demonstrated remarkably mild cellular and tissue pathology, even in the brain, despite the elevated glucocerebroside levels (19). No definite pathologic changes were observed at the light microscopic level, and typical Gaucher cells were not seen. However, electron microscopic studies revealed an accumulation of glucocerebroside within lysosomes. In the brain, the stored glucocerebroside was found predominantly in microglial cells and only to a very limited extent in certain sensory and motor neurons. Purkinje cells and neurons of the cerebellar and cerebral cortex were free of glucocerebroside (19). These findings make it difficult to attribute the early demise of the type 2 mice and human neonates solely to glucocerebroside storage.

As an alternative explanation for the rapid clinical deterioration seen in type 2 Gaucher disease, we studied the accumulation of Glc-sph in brain, liver, and spleen from mice with type 2 Gaucher disease. We also measured the level of this glycosphingolipid at different stages of gestation in affected humans and mice. On the basis of our findings, we suggest that Glc-sph rather than glucocerebroside may be responsible for the neuropathology observed in type 2 Gaucher disease.

\section{METHODS}

Chemicals. Glc-sph, Gal-sph, and NBD-F were obtained from Sigma Chemical Company (St. Louis, MO, U.S.A.).

Animals and tissue preparation. All animal studies were approved by the Animal Care and Use Committee of the
National Institute of Mental Health. A colony of heterozygous type 2 Gaucher mice (18) has been maintained in our laboratory, as well as the Jackson Laboratory mutant mouse collection. Mating between heterozygote mice produced homozygous offspring as well as heterozygous and wild-type littermates. Although homozygous mice with type 2 Gaucher disease were easily identified clinically, PCR-based genotyping was performed on DNA obtained from mouse tails. Homozygous and heterozygous mice were identified by the presence of the NeoR, which replaced portions of exons 9 and 10 of the murine glucocerebrosidase. Long PCR was performed with a forward primer from exon 9 (GAACCTCCTTTACCACGTAACTGG) and reverse primer from intron 10 (CGTGTGAGGTGGCTGGCATC) of the murine glucocerebrosidase gene using the enzyme Elongase (Life Technology, Gaithersburg, MD, U.S.A.), an annealing temperature of $57^{\circ} \mathrm{C}$, and 35 amplification cycles. Using these conditions, a 668-bp product was amplified from the wild-type allele, whereas the null glucocerebrosidase allele with NeoR resulted in a $2.1-\mathrm{kb}$ amplification product. The preparation of animal tissues was performed as described previously (19).

Human fetal tissues. Frozen autopsy tissues were received from two human fetuses affected with type 2 Gaucher disease. The diagnosis was established by both enzymatic and molecular analyses. The two fetuses were conceived by the same nonconsanguineous parents, and the pregnancies ended at 11 and $22 \mathrm{wk}$ of gestation. Both fetuses were found to have severe hydrops fetalis (21). Molecular analyses were performed on fetal and parental DNA, collected with informed consent under a protocol approved by the National Institute of Mental Health Institute Review Board. These studies revealed that the father carried a novel recombinant allele resulting from a fusion between the glucocerebrosidase gene and its pseudogene, beginning in intron 3. The maternal mutation was a rare $\mathrm{T}$ to $\mathrm{G}$ splice junction mutation in intron 10 (21). Frozen autopsy and pathologic samples from controls were purchased from the Cooperative Human Tissue Network (Philadelphia, PA, U.S.A.) and the Central Laboratory for Human Embryology (Seattle, WA, U.S.A.).

Fetal mouse tissues. Murine embryos from timed heterozygote-heterozygote matings were obtained at specific days of gestation (E10, E13, E15, E18, and E21). All littermates were genotyped, and the tissues were analyzed for Glc-sph content. Matings continued until the number of homozygous embryos at each day studied was adequate for statistical analysis. Because of the limited size of the organs in these early embryos, especially the spleen, lipid levels were measured from the head (including the brain) and were compared with levels in the rest of the body.

Glc-sph extraction and isolation. Fresh tissue was placed in a $15-\mathrm{mL}$ polypropylene tube, and approximately $100 \mathrm{mg}$ of wet weight tissue was homogenized with $5 \mathrm{~mL}$ of chloroformmethanol $(2: 1, \mathrm{vol} / \mathrm{vol})$. A $0.5-\mathrm{mL}$ aliquot was withdrawn for protein determination. For lipid analysis, the homogenate was kept at room temperature for $1 \mathrm{~h}$ and then filtered through a $0.22-\mu \mathrm{m}$ membrane (Millipore, Bedford, MA, U.S.A.). One microgram of Gal-sph was added to the filtrate as an internal standard. The lipid extract was evaporated under nitrogen to a 
volume of approximately $2 \mathrm{~mL}$ and applied to a cationic $\mathrm{AG}$ $50 \mathrm{~W}$-X8 column $(1 \times 15 \mathrm{~cm}, 1.5 \mathrm{~mL}$ bed volume, sodium form; BioRad, Richmond, CA, U.S.A.). The column was washed successively with $4 \mathrm{~mL}$ of methanol, $8 \mathrm{~mL}$ of chloroform $/ \mathrm{methanol}(2: 1, \mathrm{vol} / \mathrm{vol})$, and $4 \mathrm{~mL}$ of methanol, and then eluted with $4 \mathrm{~mL}$ of methanol-aqueous $0.4 \mathrm{M} \mathrm{CaCl}_{2}$ (3:1, $\mathrm{vol} / \mathrm{vol})$. An equal volume of water $(4 \mathrm{~mL})$ was added to the eluate, and the mixture was applied to a C18 Sep-Pak cartridge (Waters Associates, Milford, MA, U.S.A.), which was activated with methanol and washed with water before use. The cartridge was washed with $20 \mathrm{~mL}$ of water, and sphingosines were eluted with $3 \mathrm{~mL}$ of methanol, and $3 \mathrm{~mL}$ of chloroformmethanol $(2: 1, \mathrm{vol} / \mathrm{vol})$. The eluate was evaporated to dryness under nitrogen.

Sphingosine derivatization with NBD-F. Aliquots containing $10 \mu \mathrm{g}$ of both Glc-sph and Gal-sph or the nitrogen-dried sample eluate (see above) were dissolved in $100 \mu \mathrm{L}$ of methanol/0.1 M phosphate buffer ( $\mathrm{pH} 8.0,50 \% \mathrm{vol} / \mathrm{vol})$. Five microliters of NBD-F ( $80 \mathrm{mM}, 14.6 \mathrm{mg} / \mathrm{mL}$ of methanol) was added, and the mixture was heated at $70^{\circ} \mathrm{C}$ for $1 \mathrm{~min}$.

HPLC analyses of derivatized Glc-sph and Gal-sph. Aliquots of the samples were diluted 1:100 in a mobile phase $(2 \%$ water in acetonitrile) and analyzed for Glc-sph and Gal-sph by HPLC, using two 114 Solvent Delivery Modules controlled with a 421 Controller (Beckman, Fullerton, CA, U.S.A.). A Shodex Sugar column SZ $5532(5 \mathrm{~mm} \times 16 \mathrm{~cm}$, internal diameter) with a Shodex Sugar SZ precolumn (Showa Denko, Tokyo, Japan) was used for the separation of Glc-sph and Gal-sph, which was performed in acetonitrile-water (98:2, $\mathrm{vol} / \mathrm{vol}$ ) with a flow rate of $1 \mathrm{~mL} / \mathrm{min}$. The column was heated at $55^{\circ} \mathrm{C}$. A Waters 474 scanning fluorescence detector, with excitation and emission wavelengths of $470 \mathrm{~nm}$ and $530 \mathrm{~nm}$, respectively, was used to detect peaks. Data were collected for analysis, and peak areas were calculated on a Dionex chromatography data station using the Advanced Computer Interface (Dionex, Sunnyvale, CA, U.S.A.).

\section{Statistical analyses}

The significance of differences between the homozygous null, heterozygous null, and wild-type mice was analyzed using a paired $t$ test. Experimental results are expressed in the form of mean $\pm \mathrm{SD}$, and $n$ indicates the number of different mice studied.

\section{RESULTS}

Quantitation of Glc-sph. The quantitation of Glc-sph was achieved by HPLC separation of NBD-F-generated autofluorescent derivatives (22) that were detectable in the picomole range. Linearity was demonstrated over concentrations ranging 100 -fold. The efficiency of the extraction of Glc-sph from tissues was quantitated by the addition of a standard amount of Gal-sph to the samples.

Analysis of Glc-sph in tissues from mice with type 2 Gaucher disease. The separation of Glc-sph and Gal-sph (used as an internal standard) isolated from brain tissue from heterozygous and homozygous mice with type 2 Gaucher disease at birth is shown in Figure 1. Mice with type 2 Gaucher disease
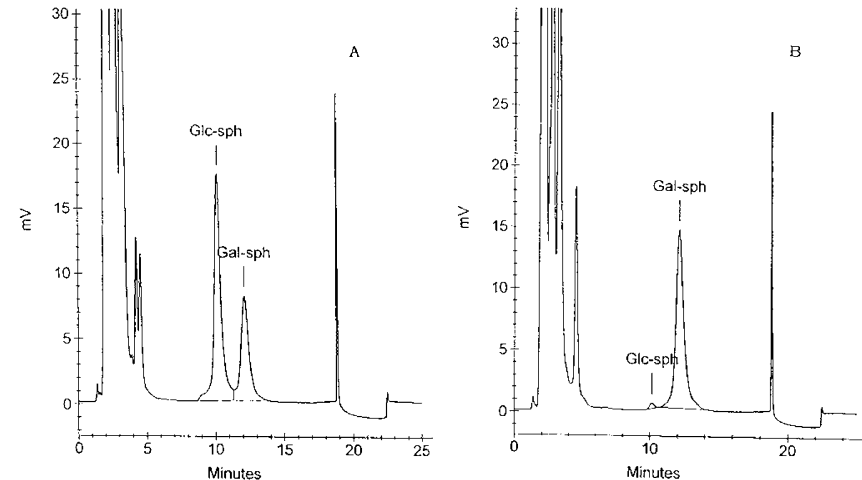

Figure 1. Quantitative profiles of Glc-sph in brain from homozygous $(A)$ and heterozygous mutant $(B)$ mice with type 2 Gaucher disease. The quantitations of Glc-sph in tissues was based on the addition of a standard amount of Gal-sph to samples as an internal standard.

accumulated 50-100 times more Glc-sph in brain (633.0 \pm 92 $\mathrm{ng} / \mathrm{mg}$ protein; $n=7$ ) compared with heterozygous (13.6 \pm 7 $\mathrm{ng} / \mathrm{mg}$ protein; $n=7)$ and wild-type (6.4 $\pm 3 \mathrm{ng} / \mathrm{mg}$ protein; $n=8)$ littermates ( $p \leq 0.0001$; Table 1). No statistical significance was found between the Glc-sph levels in heterozygous and wild-type littermates. Glc-sph levels were also markedly elevated in both liver $(555.7 \pm 59 \mathrm{ng} / \mathrm{mg}$ protein; $p=$ $0.0007)$ and spleen $(732.1 \pm 87 \mathrm{ng} / \mathrm{mg}$ protein; $p=0.0009)$ from homozygous mice with type 2 Gaucher disease (Table 1 ) compared with nonaffected littermates.

Glc-sph accumulation in embryonic mice with type 2 Gaucher disease. Early mouse embryos were studied by measuring Glc-sph in both the head portion and the remainder of the body. Glc-sph was measured at E 10, E 13, E 15, E 18, and E $21 \mathrm{~d}$ of gestation, and the numbers of homozygous null mice studied at each time point were 3, 4, 3, 3, and 7, respectively. Elevations of Glc-sph were detected in utero in mice with type 2 Gaucher disease by E13 and progressively increased throughout gestation both in the head and the body (Fig. 2). The amount of Glc-sph in control littermates was $7 \pm 4 \mathrm{ng} / \mathrm{mg}$ protein, and at least two control embryos were assayed at each gestational date.

Analysis of Glc-sph in tissues from human fetuses with type 2 Gaucher disease. Glc-sph levels were measured in different tissues from two human fetuses with type 2 Gaucher disease, received as pathology specimens at 11 and $22 \mathrm{wk}$ of gestation. The Glc-sph levels for each tissue are shown in Table 2. Significant elevations of Glc-sph were observed in both fetuses compared with tissues levels in three control fetuses (gestational ages 13 to $15 \mathrm{wk}$ ), in which Glc-sph levels were negligible (Table 2).

Table 1. Glucosylsphingosine levels in newborn mice*

\begin{tabular}{lccc}
\hline \multicolumn{1}{c}{ Tissue } & Brain & Liver & Spleen \\
\hline Wild-type control mice $(n=8)$ & $6.4 \pm 3$ & $5.0 \pm 3$ & $13.4 \pm 8$ \\
Heterozygous mice $(n=7)$ & $13.6 \pm 7$ & $3.1 \pm 3$ & $2.5 \pm 1$ \\
Homozygous mutant mice $(n=7)$ & $633.0 \pm 92$ & $555.7 \pm 59$ & $732.1 \pm 87$
\end{tabular}

* Values reported as mean $\pm \mathrm{SD}$ in $\mathrm{ng} / \mathrm{mg}$ protein. 


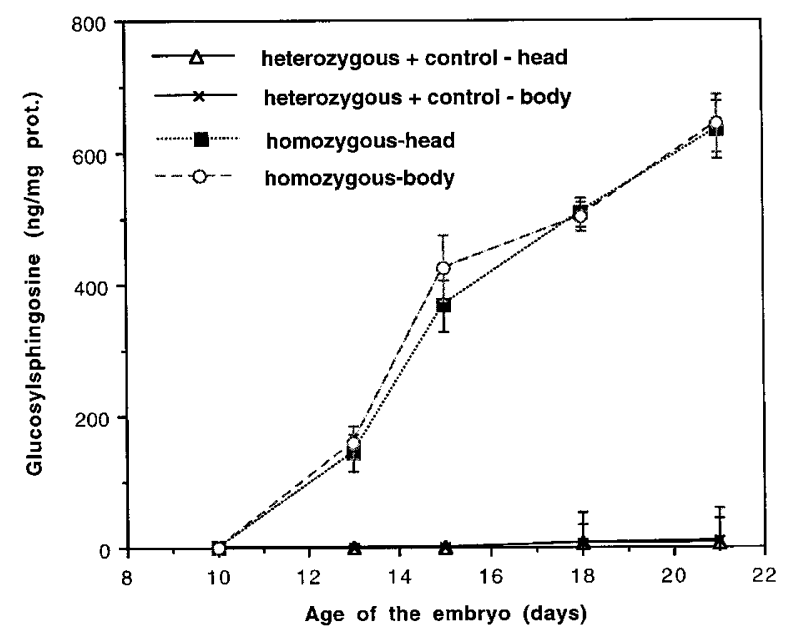

Figure 2. Glc-sph levels in murine tissue from control embryos and embryos with type 2 Gaucher disease at different gestational ages.

\section{DISCUSSION}

Many unresolved questions remain regarding both the pathophysiology of type 2 Gaucher disease and the mechanisms resulting in the wide range of phenotypic differences encountered in patients with glucocerebrosidase deficiency. Neither the levels of residual glucocerebrosidase activity, the amount of accumulated glucocerebroside, nor the PCR-defined genotype can be used to reliably predict the clinical course. The availability of a mouse model of type 2 Gaucher disease has permitted a more detailed evaluation of the biochemical and ultrastructural changes occurring in neuronopathic Gaucher disease. Like human infants with type 2 Gaucher disease, the null allele mice die in early infancy and have elevated levels of glucocerebroside in brain and other tissues. However, the limited amount of cellular pathology observed throughout the CNS suggests that the rapid demise and neurologic deterioration encountered is not solely related to glucocerebroside storage.

The average Glc-sph level in brains of newborn homozygous mice with type 2 Gaucher disease is shown to be $633.0 \pm 92$ $\mathrm{ng} / \mathrm{mg}$ protein, which is approximately 100 -fold greater than that of wild-type littermates. Similar increases in Glc-sph levels were observed in the liver and the spleen of the mice with type 2 Gaucher disease, but not in controls or heterozygotes. Although it has been suggested that chemical degradation of sphingolipids to the corresponding lyso-compound may occur under postmortem conditions (23) we were able to address this concern by simultaneously studying littermates.

The animal model also enabled us to study the accumulation of Glc-sph during development. In the mice with type 2 Gaucher disease Glc-sph was detectable in utero by E13, and Glc-sph levels progressively increased throughout gestation both in the brain and in the visceral organs.

These findings in the mice with type 2 Gaucher disease confirm and extend earlier studies performed by Nilssen and Svennerholm (15), which demonstrated that Glc-sph, which is ordinarily not detected in normal human brain, is markedly elevated in brains from patients with type 2 and 3 Gaucher disease. In fact, the highest values were reported from patient samples from the most fulminant cases. These observations suggested that the cytotoxic substance, Glc-sph, could be involved in the neuronal loss in Gaucher disease. Moreover, in another lysosomal storage disorder, Krabbe disease, the inherited deficiency of galactosylceramidase (EC 3.2.1.46), a sphingosine derivative, is directly implicated in the CNS damage observed (24). In both a naturally occurring murine model of this disorder, the Twitcher mouse, and patients with Krabbe disease, there is an accumulation of Gal-sph (galactosylpsychosine), which leads to the loss of oligodendroglia (23-25).

The in utero accumulation of Glc-sph observed in the mice with type 2 Gaucher disease was also seen in tissues from humans affected with type 2 Gaucher disease. The lipid determinations performed on tissues obtained from two fetuses, both diagnosed prenatally with type 2 Gaucher disease, reveal Glc-sph storage occurring as early as $11 \mathrm{wk}$ of gestation. The levels of Glc-sph in the human fetuses were quite close to those of mice at comparable stages of gestation. Compared with Glc-sph levels in tissue from control human fetuses, the Glcsph levels in the fetuses with type 2 Gaucher disease suggest advanced and progressive accumulation analogous to the storage observed in mice with type 2 Gaucher disease. Clinically, these findings have important implications. They suggest that a toxic stimulus is present long before birth, and that therapy begun during infancy may do little to prevent the progressive neurologic damage that began early in gestation.

We have measured brain Glc-sph levels in seven patients with Gaucher disease (26). Five of the patients studied had type 2 Gaucher disease, and the levels of Glc-sph ranged from 9.8 to $935 \mathrm{ng} / \mathrm{mg}$ protein. A patient with type 3 Gaucher disease had a Glc-sph level of $8.9 \mathrm{ng} / \mathrm{mg}$ protein. The brain Glc-sph level, measured in one adult patient with type 1 Gaucher disease who died at age 69 years, was $0.34 \mathrm{ng} / \mathrm{mg}$ protein, which was in the range detected in six control adult brain samples $(0.1-1.4 \mathrm{ng} / \mathrm{mg}$ protein, mean $0.6 \mathrm{ng} / \mathrm{mg}$ protein). In the literature, the brain Glc-sph level was quantified in a single patient who died at age 13 years and was believed to have type 1 Gaucher disease (27). Here the concentration of Glc-sph in the cerebral cortex was reported to be $0.7 \mathrm{nmole} / \mathrm{g}$, which was higher than the controls $(<0.01 \mathrm{nmole} / \mathrm{g})$ but well below values that they reported for their patients with type 2 Gaucher disease (3.9-14.8 nmole/g) (27).

It is now appreciated that glycolipids, once thought to serve only as structural components of cells, are important in signal pathways, cellular senescence, and programmed cell death (16, 28). Studies have shown that Glc-sph can inhibit the growth of certain cells in culture (29). Glc-sph, as well as Gal-sph and sphingosine, is a potent inhibitor of mitochondrial cytochrome $c$ oxidase (30) and protein kinase $\mathrm{C}$ activity $(16,28)$, and could disrupt cell activity by interfering with signal transduction and cellular differentiation. Furthermore, both sphingosine and ceramides have been implicated as mediators of apoptosis (17). Thus, the Glc-sph accumulation noted in patients and mice with type 2 Gaucher disease may have far-reaching effects. Studies are currently under way to better establish a mechanistic basis for the cellular toxicity of Glc-sph. 
Table 2. Fetal glucosylsphingosine levels

\begin{tabular}{|c|c|c|c|c|c|}
\hline Affected Status & Gaucher disease & Gaucher disease & Control & Control & Control \\
\hline Gestational age (wk) & 11 & 22 & 13 & 14 & 15 \\
\hline \multicolumn{6}{|c|}{ Glucosylsphingosine level (ng/mg protein) } \\
\hline Brain & 304.8 & 437.0 & 0.3 & 0.1 & 0.04 \\
\hline Spleen & NA & 189.9 & NA & 0.2 & 0.1 \\
\hline
\end{tabular}

NA, not available.

Our current results provide further evidence that Glc-sph may be involved in the cause of the neuronopathic forms of Gaucher disease because 1) brain Glc-sph is markedly elevated in mice with type 2 Gaucher disease, 2) Glc-sph is also elevated in tissues including brain from human fetuses with type 2 Gaucher disease, and 3) mice with type 2 Gaucher disease progressively accumulate Glc-sph throughout gestation. Although the accumulation of both glucocerebroside and Glc-sph in patients with Gaucher disease is a direct consequence of glucocerebrosidase deficiency, the origin of the stored Glc-sph and its metabolism still require better characterization. The accumulation of Glc-sph during embryonic development could result in irreversible damage to the developing nervous system and lead to the devastating clinical course seen in mice and patients with type 2 Gaucher disease.

Acknowledgments. The authors thank Lester Carmon for technical assistance with animal procedures and Drs. Evelyn Carson and Rome Chandra for their clinical and pathologic contributions. We also thank Elizabeth Alzona and Kay Kuhns for their secretarial assistance.

\section{REFERENCES}

1. Gaucher PCE 1882 De l'epithelioma primitif de la rate, hypertrophie idiopathique de la rate sans leucemie. Faculte de Medecine, These de Paris 31

2. Aghion H 1934 La Maladie de Gaucher dans l'enfance. Faculte de Medecine, These de Paris

3. Brady RO, Kanfer JN, Shapiro D 1965 Metabolism of glucocerebrosides II: evidence of an enzymatic deficiency in Gaucher's disease. Biochem Biophys Res Commun 18:221-225

4. Rosenberg A, Chargaff E 1958 A reinvestigation of the cerebroside deposited in Gaucher's disease. J Biol Chem 233:1323-1326

5. Grafe M, Thomas C, Schneider J, Katz B, Wiley C 1988 Infantile Gaucher's disease: a case with neuronal storage. Ann Neurol 23:300-303

6. Erickson A, Bambi B, Schiffman R 1997 Neuronopathic forms of Gaucher's disease. Baillieres Clin Haematol 10:711-723

7. Gatt S 1966 Enzymatic hydrolysis of sphingolipids. I. Hydrolysis and synthesis of ceramides by an enzyme from rat brain. J Biol Chem 25:3724-3730

8. Bernardo K, Hurwitz R, Zenk T, Desnick RJ, Ferlinz K, Schuchman EH, Sandhoff K 1995 Purification, characterization, and biosynthesis of human acid ceramidase. J Biol Chem 270:11098-11102

9. Frederickson DS, Sloan HR 1972 Glucosylceramide lipidoses: Gaucher's disease. In: Stanbury JB, Wyngaarden JB, Frederickson S (eds) The Metabolic Basis of Inherited Disease, 3rd Ed. McGraw-Hill, New York, pp 730-759

10. Sidransky E 1997 New perspectives in type 2 Gaucher disease. Adv Pediatr 44:73-
11. Lee RE 1982 Pathology of Gaucher disease. In: Desnick RJ, Gatt S, Grabowski GA (eds) Gaucher Disease: A Century of Delineation and Research. Alan R. Liss, New York, pp 177-217

12. Barranger JA, Ginns EI 1989 Glucosylceramide lipidoses: Gaucher disease. In: Scriver CR, Beaudet AL, Sly WS, Valle D (eds) The Metabolic Basis of Inherited Disease, 6th Ed. McGraw-Hill, New York, pp 1677-1698

13. Grabowski GA 1997 Gaucher disease: gene frequencies and genotype/phenotype correlations. Genet Test 1:5-12

14. Sidransky E, Bottler A, Stubblefield B, Ginns EI 1994 DNA mutational analysis of type 1 and type 3 Gaucher patients: how well do mutations predict phenotype? Hum Mutat 3:25-28

15. Nilsson O, Svennerholm L 1982 Accumulation of glucosylceramide and glucosylsphingosine (psychosine) in cerebrum and cerebellum in infantile and juvenile Gaucher disease. J Neurochem 39:709-718

16. Hannun YA, Bell RM 1987 Lysosphingolipids inhibit protein kinase C: implications for the sphingolipidoses. Science 235:670-675

17. Speigel S, Merrill AH 1996 Sphingolipid metabolism and cell growth regulation. FASEB J 120:1388-1397

18. Tybulewicz VLJ, Tremblay ML, LaMarca ME, Willemsen R, Stubblefield BK, Winfield S, Zablocka B, Sidransky E, Martin BM, Huang SP, Mintzer KA, Westphal H, Mulligan RC, Ginns EI 1992 Animal model of Gaucher's disease from targeted disruption of the mouse glucocerebrosidase gene. Nature 357:407-412

19. Willemsen R, Tybulewicz V, Sidransky E, Eliason WK, Martin BM, LaMarca ME, Reuser AJJ, Tremblay M, Westphal H, Mulligan RC, Ginns EI 1995 A biochemical and ultrastructural evaluation of the type 2 Gaucher mouse. Mol Chem Neuropathol 24:179-192

20. McKinney CE, Sidransky E, LaMarca ME, Riviere I, Holleran WM, Martin BM, Willemsen R, Mulligan RC, Ginns EI 1995 Gaucher disease: a tale of two species. Ment Retard Dev Disabil Res Rev 1:79-86

21. Reissner K, Tayebi N, Stubblefield BK, Blitzer M, Holleran W, Cowan T, Almashanu S, Maddalena A, Karson EM, Sidransky E 1998 Type 2 Gaucher disease with hydrops fetalis in an Ashkenazi Jewish family resulting from a novel recombinant allele and a rare splice junction mutation in the glucocerebrosidase locus. Mol Genet Metab 63:281-288

22. Nozawa M, Iwamoto T, Tokoro T, Eto Y 1992 Novel procedure for measuring psychosine derivatives by an HPLC method. J Neurochem 59:607-609

23. Miyatake T, Suzuki K 1972 Globoid cell leukodystrophy: additional deficiency of psychosine galactosidase. Biochem Biophys Res Commun 48:538-543

24. Suzuki K 1998 Twenty five years of the "Psychosine Hypothesis": a personal perspective of its history and present status. Neurochem Res 23:251-259

25. Kobayashi T, Shinoda H, Goto I, Yamanaka T, Suzuki Y 1987 Globoid cell leukodystrophy is a generalized galactosylsphingosine (psychosine) storage disease. Biochem Biophys Res Commun 144:41-46

26. Orvisky E, Ginns EI, Sidransky E 1999 Glucosylsphingosine accumulation in patients with Gaucher disease. Am J Hum Genet 65:A427

27. Svennerholm L, Hakansson G, Mansson JE, Nilsson O 1982 Chemical differentiation of the Gaucher subtypes. In: Desnick RJ, Gatt S, Grabowski GA (eds) Gaucher Disease: A Century of Delineation and Research. Alan R. Liss, New York, pp 231-252

28. Hannun YA 1994 The sphingomyelin cycle and the second messenger function of ceramide. J Biol Chem 269:3125-3128

29. Atsumi S, Nosaka C, Iinuma H, Umezawa K 1993 Accumulation of tissue glucosylsphingosine in Gaucher-like mouse induced by the glucosylceramidase inhibitor cyclophellitol. Arch Biochem Biophys 1:302-304

30. Igisu H, Hamasaki N, Ito A, Ou W 1988 Inhibition of cytochrome $c$ oxidase and hemolysis caused by lysosphingolipids. Lipids 23:345-348 